\title{
Shock and Blast Interaction with Thin Films
}

\author{
A.J. Higgins \\ McGill University, 817 Sherbrooke St. West, Montreal, H3A 0C3, Canada \\ Corresponding Author: andrew.higgins@mcgill.ca
}

The interaction of a shock wave with a density interface is a well-studied problem as the Richtmyer-Meshkov (RM) instability [Brouillette 2018, Zhai et al. 2018]. In RM studies, the presence of a thin film used to separate the two gases is usually viewed as an experimental inconvenience. In this study, the role of the film in suppressing instability is specifically examined. In particular, the influence of film thickness and pretensioning of the development of instability is observed for both supported shock waves generated in a shock tube and decaying shock waves (blast waves) of different strength.

For the shock-tube driven loading, a conventional $12.7 \mathrm{~cm}$ by $12.7 \mathrm{~cm}$ square crosssectional shock tube is used, and the thin film is pre-tensioned using conventional shock tube diaphragm techniques. For blast-wave loading, a commercial explosive transfer tube (Nonel or "shock tube") is used. The blast wave emanating from the end of Nonel with $20 \mathrm{mg} / \mathrm{m}$ of explosive loading has been previously characterized has having $\sim 1 \mathrm{~J}$ of blast energy [Samuelraj et al. 2013]. The Nonel was directed at the back side of the film and observed from the front. The dynamics of the film is recorded by use of high-speed videography (Photron SA-5) and photonic Doppler velocimetry (PDV), as shown schematically in Fig. 1. The downstream side of the film (blast impingement side) could be exposed to different gaseous environments (helium, etc.) by flushing the volume between the end of the Nonel tube in order to vary the Atwood number of the blast loading.

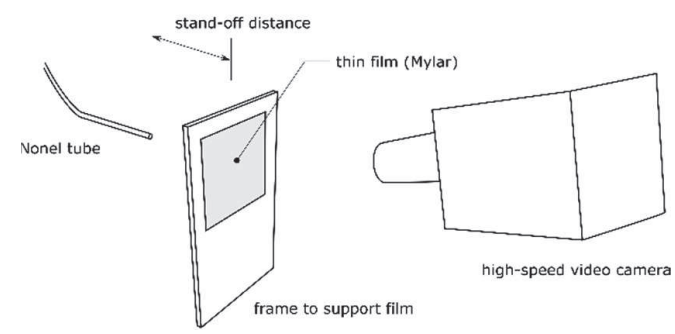

Figure 1. Schematic of experimental set-up for blast interaction with thin films.

Sample results are shown here for blast wave loading of aluminum-coated polyester film (Mylar) in Fig. 2. For a 2- $\mu \mathrm{m}$ film that is nonpretensioned, surface undulations appear within $10 \mu \mathrm{s}$ of shock impingement with a sub-mm characteristic wavelength scale. As the Mylar film is made thicker $(6 \mu \mathrm{m})$, the scale of instability increases to mm-scale wavelength. For even thicker film $(12 \mu \mathrm{m})$, instability is suppressed entirely. Even for the thinnest films tested, in all cases, eventual bulk outward motion (bowing) of the film results in the diaphragm experiencing a state of tension that suppresses instability at approximately $200 \mu$ s after shock impingement. These results suggest a trade-off between film thickness and tension that can suppress the inherent instability of shock-accelerated interfaces.
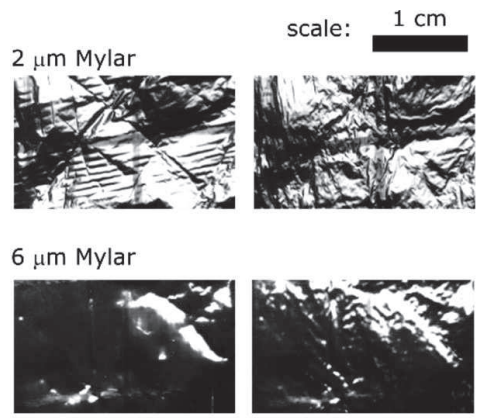

$12 \mu \mathrm{m}$ Mylar

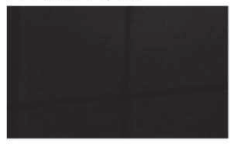

$0 \mu \mathrm{s}$ (shock arrival)

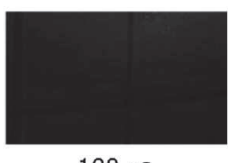

Figure 2. Sample of results of blast interaction with thin polyester (Mylar) film.

The results have consequences for understanding the influence of the early stage of diaphragm rupture on shock waves and subsequent shock-driven flows in unsteady facilities in which shock waves must traverse diaphragms (shocktunnels and expansion tunnels, etc.). The understanding of how materials strength effects can suppress RM and RT instability has applications to inertial confinement [Nellis 2009] and magnetized target fusion. This particular study is motivated by the need to test how very thin films (lightsails, that can be as thin as $200 \mathrm{~nm}$ ) respond to dynamic loads as would be encountered in laserdriven relativistic spaceflight [Lubin 2016]. 


\section{References}

Brouillette, M.: The Richtmyer-Meshkov instability. Annual Review of Fluid Mechanics 34(1), 445-468 (2002).

Lubin, P.: A Roadmap to Interstellar Flight, Journal of the British Interplanetary Society, 69, 40-72 (2016).

Nellis,W.J.: Will NIF Work? (2009) https://arxiv.org/abs/0908.4229

Samuelraj, I.O., Jagadeesh, G., and Kontis, K. Micro-blast waves using detonation transmission tubing. Shock Waves 23: 307 (2013).

Zhai, Z., Zou, L., Wu, Q., and Luo, X.: Review of experimental Richtmyer-Meshkov instability in shock tube: From simple to complex. Proceedings of the Institution of Mechanical Engineers, Part C: Journal of Mechanical Engineering Science 232(16), 2830-2849 (2018). 